\title{
Descriptive review of clinical data from 186 records of outpatients with IgA deficiency accompanied at a quaternary hospital in Brazil
}

\author{
Fabiana Mascarenhas ${ }^{1 *}$, Myrthes Toledo Barros², Leonardo Mendonça' ${ }^{1}$ Cristina Kokron ${ }^{3}$, Karla Boufleur ${ }^{1}$, \\ Pablo Torres ${ }^{1}$, Ana Karolina Barreto De Oliveira ${ }^{4}$, Octavio Grecco ${ }^{1}$, Jorge Kalil ${ }^{3}$, Andrea Cohon ${ }^{5}$
}

From 3rd WAO International Scientific Conference (WISC) 2014

Rio de Janeiro, Brazil. 6-9 December 2014

\section{Background}

IgA Deficiency (IgAD) is the most common immunoglobulin deficiency, with approximate incidence of 1 in 400 to 3000 individuals in general population. Selective IgA deficiency is defined as serum IgA levels less than $7 \mathrm{mg} / \mathrm{dL}$ with normal serum IgM and IgG levels. A subgroup of individuals, with more than $7 \mathrm{mg} / \mathrm{dL}$ but less than $30 \mathrm{mg} / \mathrm{dL}$ of serum IgA, are defined as partial IgA deficient patients (pIgAD). Many individuals with selective IgA deficiency are clinically asymptomatic. However, some patients have increased incidence of infections, mainly in respiratory and intestinal tracts, as well as presence of atopy, autoimmunity and cancer.

\section{Methods}

Records review from children and adults outpatients followed at the Clinical Immunology and Allergy Division at Hospital das Clinicas of FMUSP. All patients were orally informed about the use of these clinical data. The objective was to report the clinical data observed in a cohort of IgAD patients followed between 1994 and 2014.

\section{Results}

The most common associated condition found was atopic disease in about $60 \%$ of patients, specially rhinitis (in tIgAD and PIgAD). Prevalence of recurrent infection was $36 \%: 20 \%$ of sinusitis, otitis and amigdalitis, $10 \%$ of recurrent pneumonia (more than twice in the last year) and $18 \%$ of recurrent diarrhea. Autoimmunity condition was observed in $18 \%$ of the patients. Autoimmune diseases detected were thyroiditis, autoimmune hemolytic anemia, idiopathic thrombocytopenic purpura, rheumatoid arthritis, celiac-like disease, systemic lupus, Sjögren's disease, juvenile rheumatoid arthritis, autoimmune hepatitis and vitiligo. The most prevalent disease observed was thyroid disease in about $13 \%$ of the patients. History of medication reaction was observed in $9 \%$ of them and contact dermititis in $8 \%$. About $9 \%$ of the IgAD patients had malignancies or pre neoplastic conditions like MGUS (monoclonal gamopathy with uncertain signifcance). It was not possible to establish rate mortality among these patients because many patients lost follow up for unknown reasons, but so far, only one death was reported, and was attributed to sepsis.

\section{Conclusions}

Total and partial IgAD has a broad spectrum of clinical associations and further investigations to immunological intervention may have clinical results.

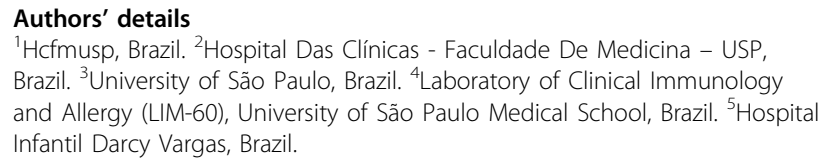
Brazil. ${ }^{3}$ University of São Paulo, Brazil. ${ }^{4}$ Laboratory of Clinical Immunology and Allergy (LIM-60), University of São Paulo Medical School, Brazil. ${ }^{5}$ Hospital Infantil Darcy Vargas, Brazil.

Published: 8 April 2015

\section{doi:10.1186/1939-4551-8-S1-A162}

Cite this article as: Mascarenhas et al: Descriptive review of clinical data from 186 records of outpatients with IgA deficiency accompanied at a quaternary hospital in Brazil. World Allergy Organization Journal 2015 8(Suppl 1):A162. 